\title{
Exotic branes in Double Field Theory
}

\author{
Edvard Musaev ${ }^{12, \star}$ \\ ${ }^{1}$ Max-Planck-Institut für Gravitationsphysik (Albert-Einstein-Institut), Am Mühlenberg 1, DE-14476 Potsdam, \\ Germany \\ ${ }^{2}$ Kazan Federal University, Institute of Physics, General Relativity Department, Kremlevskaya 16a, 420111, \\ Kazan, Russia
}

\begin{abstract}
The non-geometric Q- and R-monopole are shown to be a particular case of the DFT-monopole solution. The notion of magnetic charge for the solutions is defined and shown to be equal to the magnetic charge of the NS5-brane solution. This is a talk presented by the author at the conference QUARKS' 16 in St.-Petersburg.
\end{abstract}

\section{T-duality and Double Field Theory}

It is known that string theory respects a hidden symmetry, called T-duality, on the quantum perturbative level. The most intuitive way of understanding the action of this symmetry is consider swapping of momentum and winding modes of a closed string on a $d$-torus $\mathbb{T}^{d}$. In this case the T-duality symmetry group is $O(d, d)$. It has long been conjectured that these symmetries must carry some geometric meaning which would allow to define them on non-toroidal backgrounds.

The recently constructed $O(d, d)$-covariant gravity-like theory called Double Field Theory exploits the old ideas by Tseytlin [1] and Siegel [2] that left and right movers on the string worldsheet should be considered independently and generalizes the construction of generalized geometry by Hitchin, Cavalcanti and Gualtiery (see [3] for review and further links). The pioneering works in this direction were $[4,5]$ by Hull, Hohm and Zwiebach who presented the background independent duality covariant formulation of the action of DFT in terms of the so-called generalized metric $\mathcal{H}_{M N}$. Soon after the same was done for the U-duality groups in [6-8] for the internal sector only, and in [9-12] for the full theory including $p$-forms. Supersymmetric extension and inclusion of fermions has been presented in $[13,14]$ for DFT and in $[15,16]$ for EFT.

The idea behind Double Field Theory is that one doubles the space-time by adding dual coordinates $\tilde{x}_{\mu}$ corresponding to winding modes of closed strings. The resulting space-time (better to say 'space' to avoid issues with imaginary time) is parametrized by $(d+d)$ coordinates $\mathbb{X}^{M}=\left\{x^{\mu}, \tilde{x}_{\mu}\right\}$. The fields of the NS-NS sector, i.e. the metric, the B-field, and the dilaton, combine into the generalized metric $\mathcal{H}_{M N}$ that transforms covariantly under $O(d, d)$ transformations $O$ and depends on all the coordinates

$$
\mathcal{H}_{M N}=\left[\begin{array}{cc}
g-B g^{-1} B & g^{-1} B \\
B g^{-1} & g^{-1}
\end{array}\right], \quad \mathcal{H} \rightarrow O^{T} \mathcal{H} O
$$

^e-mail: edvard.musaev@aei.mpg.de 
By an $O(d) \times O(d)$ rotation this can equivalently be parametrized by the metric and a bivector field $\beta$, that is useful for defining non-geometric fluxes

$$
\mathcal{H}_{M N}=\left[\begin{array}{cc}
g & \beta g^{-1} \\
g^{-1} \beta & g^{-1}-\beta g^{-1} \beta
\end{array}\right] .
$$

Locally T-duality transformations are represented by generalized Lie derivative that combines these with conventional diffeomorphisms and gauge transformations of the B-field, and is defined by its action on a generalized vector $V^{M}$ along a generalized vector $U^{M}$

$$
\mathcal{L}_{U} V^{M}=U^{N} \partial_{N} V^{M}-V^{N} \partial_{N} U^{M}+\eta^{M N} \eta_{K L} \partial_{N} U^{K} V^{L},
$$

where $\eta_{M N}$ is the (skew-diagonal) invariant tensor of $O(d, d)$. Algebra of these transformations generalizes that of the Dorfmann brackets and closes on the so-called C-bracket, that generalizes the Courant bracket, upon a special constraint

$$
\begin{aligned}
{\left[\mathcal{L}_{V_{1}}, \mathcal{L}_{V_{2}}\right] } & =\mathcal{L}_{\left[V_{1}, V_{2}\right]_{C}}+F_{0}, \\
\operatorname{Jac}\left(\mathcal{L}_{V_{1}}, \mathcal{L}_{V_{2}}, \mathcal{L}_{V_{3}}\right) & =F_{1} .
\end{aligned}
$$

Here the additional terms $F_{0}$ and $F_{1}$ represent breaking of the closure and Jacobi conditions, and vanish upon setting

$$
\eta^{M N} \partial_{M} \Phi \partial_{N} \Psi=0
$$

for any fields $\Phi, \Psi$. This constraint called the section condition effectively reduces the number of coordinates to $d$ and returns the dynamics back to the conventional supergravity.

Given this equivalence there has been a lot of discussion in the community whether DFT is just a more convenient rewriting of supergravity or it may actually give new results. The results discussed below suggest that DFT (and similarly EFT) is indeed able in principle to capture dynamics of non-geometric backgrounds. One of the ways of doing that is to consider backgrounds with fields satisfying the section condition, but which depend explicitly on dual (winding) coordinates. These have no description in the framework of the conventional supergravity as it does not know anything about the dual coordinates. Hence, these are purely DFT backgrounds, some particular examples of which will be shown to carry non-geometric flux. In what follows we briefly describe the results of [17] sending those interested in the technical details to the original paper.

\section{Exotic branes in string theory}

Probably the most famous example of non-geometric fluxes is given by $Q$ - and $R$-fluxes, which are known to correspond to non-commutative and non-associative behavior of strings respectively. These appear as nodes of the T-duality orbit starting from the geometric flux $H=d B$. Hence, one writes

$$
H_{x y z} \stackrel{T_{x}}{\longrightarrow} \tau_{y z}^{x} \stackrel{T_{y}}{\longrightarrow} Q^{x y} \stackrel{T_{z}}{\longrightarrow} R^{x y z},
$$

where $\tau^{x}{ }_{y z}$ is the so-called geometric flux, related to curvature of the background. Existence of this orbit was conjecture in [18] where a simple toy model of twisted torus has been considered. Here we would like to proceed with realistic backgrounds starting with NS5-brane with non-zero H-flux. This is written in terms of the harmonic function $H=H\left(x^{1}, \ldots, x^{d}\right)$ that is a solution of the (flat) Laplace equation with a Dirac-delta-like source term in the RHS

$$
\left(\partial_{1}^{2}+\cdots+\partial_{d}^{2}\right) H\left(x^{1}, \ldots, x^{d}\right) \sim \delta\left(x^{1}, \ldots, x^{d}\right)
$$


In what follows we will not take into account the source term and focus only on the vacuum solution, however an extensive discussion of the issue of sources for geometric and non-geometric solutions can be found in [19]. The harmonic function

$$
H\left(x^{1}, \ldots, x^{d}\right)=1+\frac{h_{0}}{\left(x_{1}^{2}+\ldots+x_{d}^{2}\right)^{\frac{d-2}{2}}},
$$

where for $d=2$ the power function should be replaced by the log and hence is not well defined. This is the well known issue of the non-geometric $5_{2}^{2}$-brane, carrying $Q$-flux (see [20] for discussion).

Table 1. Under T-dualities an NS5-brane stretched in directions marked by $\times$ turns into a Kaluza-Klein monopole and a $5_{2}^{2}$-brane. Dotted circles denote special cycles along which the T-duality acts, these are compactified.

\begin{tabular}{|r|cccc|ccccc|}
\hline & 1 & 2 & 3 & 4 & 5 & 6 & 7 & 8 & 9 \\
\hline NS5 & $\cdot$ & $\cdot$ & $\cdot$ & $\cdot$ & $\times$ & $\times$ & $\times$ & $\times$ & $\times$ \\
KKM & $\cdot$ & $\cdot$ & $\cdot$ & $\odot$ & $\times$ & $\times$ & $\times$ & $\times$ & $\times$ \\
52 & $\cdot$ & $\cdot$ & $\odot$ & $\odot$ & $\times$ & $\times$ & $\times$ & $\times$ & $\times$ \\
\hline
\end{tabular}

To act by a T-duality along an isometry direction of the NS5-brane solution one should first smear the brane along, say, $x^{4}=: z$, that is equivalent to compactification of this direction. I.e. the background with smeared harmonic function is a solution of the theory compatified on a circle [21]

$$
\frac{h_{0}}{\left(x_{1}^{2}+\ldots+x_{d}^{2}\right)^{\frac{d-2}{2}}} \Longrightarrow \frac{2 \pi R_{d} h_{0}}{\left(x_{1}^{2}+\ldots+x_{d-1}^{2}\right)^{\frac{d-3}{2}}}
$$

and the resulting background is that of the Kaluza-Klein monopole

$$
\begin{aligned}
& d s^{2}=d s_{056789}^{2}+H\left(d \rho^{2}+\rho^{2} d \theta^{2}+\left(d x^{3}\right)^{2}\right)+H^{-1}\left(d x^{4}+\omega\right)^{2}, \\
& B^{(2)}=0, \quad \rho^{2}=\left(x^{1}\right)^{2}+\left(x^{2}\right)^{2} .
\end{aligned}
$$

This solution is characterized by the geometric flux $\tau_{m n}{ }^{k}$ defined as a structure constant of the algebra of right-invariant vector fields (vielbeins) $\left[e_{m}, e_{n}\right]=\tau_{m n}{ }^{k} e_{k}$.

Smearing along another direction, say $x^{3}$, and performing T-duality $T_{x^{3}}$ one arrives to a nongeometric background usually referred to as $5_{2}^{2}$-brane [20]

$$
\begin{aligned}
& d s^{2}=H\left(d \rho^{2}+\rho^{2} d \theta^{2}\right)+\frac{H}{H^{2}+\sigma^{2} \theta^{2}} d s_{34}^{2}+d s_{056789}^{2}, \\
& B^{(2)}=\frac{\sigma \theta}{H^{2}+\sigma^{2} \theta^{2}} d x^{3} \wedge d x^{4}, \quad e^{-2 \phi}=\frac{H}{H^{2}+\sigma^{2} \theta^{2}} .
\end{aligned}
$$

This solution was constructed in [20] as a realistic example of the toy background of [18], which was conjectured to carry a portion of $Q$-flux. The most interesting property of the solution is the non-trivial monodromy around the brane $\theta \rightarrow \theta+2 \pi$ that mixes the metric and the B-field acting as a T-duality transformation on the torus $\left(x^{3}, x^{4}\right)$. Namely, the size of the special 2-torus does not come back to itself

$$
\begin{aligned}
\theta=0: & G_{33}=G_{44}=H^{-1}, \\
\theta=2 \pi: & G_{33}=G_{44}=\frac{H}{H^{2}+(2 \pi \sigma)^{2}} .
\end{aligned}
$$


In the DFT notation the monodromy takes the form of an $O(2,2)$ rotation acting as

$$
\mathcal{H}\left(\theta^{\prime}=\theta+2 \pi\right)=O^{\operatorname{tr}} \mathcal{H}(\theta) O, \quad O=\left[\begin{array}{cc}
\mathbf{1}_{\mathbf{2}} & 0 \\
\beta\left(\theta^{\prime}\right) & \mathbf{1}_{\mathbf{2}}
\end{array}\right],
$$

where $\beta\left(\theta^{\prime}\right)=\beta\left(\theta^{\prime}\right)^{34} \partial_{3} \wedge \partial_{4}$ is a bivector.

Hence, the background is defined globally only up to a T-duality transformation and in principle could be described by the T-fold construction. However, formally performing T-duality along the remaining coordinate we arrive to a background, which carry a portion of R-flux and which cannot be described by supergravity even locally. To deal with such kind of backgrounds and to define T-duality along a non-isometry direction one has to use the formalism of Double Field Theory.

\section{Exotic objects in DFT}

\subsection{DFT monopole}

Dynamics of DFT is encoded by the following effective potential

$$
V=e^{-2 d}\left(\frac{1}{8} \mathcal{H}^{M N} \partial_{M} \mathcal{H}^{K L} \partial_{N} \mathcal{H}_{K L}-\frac{1}{2} \mathcal{H}^{K L} \partial_{L} \mathcal{H}^{M N} \partial_{N} \mathcal{H}_{K M}-2 \partial_{M} d \partial_{N} \mathcal{H}^{M N}+4 \mathcal{H}^{M N} \partial_{M} d \partial_{N} d\right)
$$

which drops back to the bosonic Lagrangian density of supergravity upon the section condition. In [22] a solution of DFT equations of motion called DFT-monopole has been found. To write the solution it is convenient to understand the generalized metric as a Kaluza-Klein decomposition and to write the following DFT interval

$$
d s_{D F T}^{2}=\left(g_{\mu v}-B_{\mu}{ }^{\rho} B_{\rho v}\right) d x^{\mu} d x^{v}+2 B_{\mu}^{v} d x^{\mu} d \tilde{x}_{v}+g^{\mu v} d \tilde{x}_{\mu} d \tilde{x}_{v} .
$$

One should note that this is just a notation and the above quantity in principle does not have to indeed measure distance in the extended space.

With that in hands one writes the solution in the Taub-NUT form as follows

$$
\begin{aligned}
d s_{D F T}^{2} & =H\left(1+H^{-2} A^{2}\right) d z^{2}+H^{-1} d \tilde{z}^{2}+2 H^{-1} A_{i}\left(d y^{i} d \tilde{z}-\delta^{i j} d \tilde{y}_{j} d z\right) \\
& +H\left(\delta_{i j}+H^{-2} A_{i} A_{j}\right) d y^{i} d y^{j}+H^{-1} \delta^{i j} d \tilde{y}_{i} d \tilde{y}_{j} \\
& +\eta_{r s} d x^{r} d x^{s}+\eta^{r s} d \tilde{x}_{r} d \tilde{x}_{s},
\end{aligned}
$$

where the functions $H, A_{i}$ and the invariant dilation are given by $(i, j, k, l=1,2,3)$

$$
\begin{aligned}
H(y) & =1+\frac{h}{\sqrt{\delta_{i j} y^{i} y^{j}}}, \\
2 \partial_{[i} A_{j]} & =\epsilon_{i j k} \partial_{k} H, \\
e^{-2 d} & =H e^{-2 \varphi_{0}} .
\end{aligned}
$$

The coordinates $\left(x^{r}\right)$ with $r, s$ running from 5 to 9 and 0 , labeling the remaining directions; in addition we keep the notation $z=x^{4}$.

The solution depends on 20 parameters $\left(z, y^{i}, x^{r}, \tilde{z}, \tilde{y}_{i}, \tilde{x}_{r}\right)$, which in principle can be identified with either physical or dual coordinates of DFT. This is equivalent to choosing a T-duality frame or performing a T-duality transformation. The important difference here is, that these coordinates do not have to be compact. 
The most intriguing feature of the DFT-monopole solution is that it combines the three backgrounds of Type II supergravity, $\mathrm{H}$-, KK-monopoles and the $5_{2}^{2}$-brane, and the locally non-geometric background of R-brane upon a choice of a solution of the section condition. While the behaviour of string on the background of $5_{2}^{2}$-brane is known to be non-commutative, the R-brane background was conjectured to show signs of non-associativity. Indeed, commutator and Jacobiator of string coordinates on backgrounds with Q- and/or R-fluxes can be written as (see e.g. [23])

$$
\begin{aligned}
{\left[x^{m}, x^{n}\right] } & =Q_{k}^{m n} x^{k}, \\
{\left[x^{m}, x^{n}, x^{k}\right] } & =R^{m n k} .
\end{aligned}
$$

Since a solution of the section condition is determined by a choice of physical and dual coordinates, one may write the following general rule for embedding of the backgrounds with $\mathrm{H}, \tau, \mathrm{Q}$ and $\mathrm{R}$ fluxes into the DFT-monopole solution

Table 2. Rules for identification of the parameters $\left(z, y^{i}, \tilde{z}, \tilde{y}_{i}\right)$ with the physical coordinates $\left(x^{z}, x^{1}, x^{2}, x^{3}\right)$. The remaining parameters are identified with the dual coordinates.

$$
\begin{array}{ll}
\left(x^{z}, x^{1}, x^{2}, x^{3}\right)=\left(z, y^{1}, y^{2}, y^{3}\right), & \text { NS5-brane (H-monopole) } \\
\left(x^{z}, x^{1}, x^{2}, x^{3}\right)=\left(\tilde{z}, y^{1}, y^{2}, y^{3}\right), & \text { KK-monopole } \\
\left(x^{z}, x^{1}, x^{2}, x^{3}\right)=\left(\tilde{z}, y^{1}, y^{2}, \tilde{y}^{3}\right), & \text { Q-monopole } \\
\left(x^{z}, x^{1}, x^{2}, x^{3}\right)=\left(\tilde{z}, \tilde{y}^{1}, \tilde{y}^{2}, y^{3}\right), & \text { R-monopole. }
\end{array}
$$

Definition of fluxes in DFT in similar to that of the geometric flux $\tau_{m n}{ }^{k}$ in supergravity, i.e. these are structure constants of the algebra of generalized vielbeins $E_{M}{ }^{A}$

$$
\begin{aligned}
\mathcal{H}_{M N} & =E_{M}{ }^{\bar{A}} E_{M}{ }_{M}^{\bar{B}} \mathcal{H}_{\bar{A} \bar{B}}, \\
{\left[E_{\bar{A}}, E_{\bar{B}}\right]_{C} } & =\mathcal{F}_{\bar{A} \bar{B}}{ }^{\bar{C}} E_{\bar{C}} .
\end{aligned}
$$

Components of the generalized flux $\mathcal{F}_{\bar{A} \bar{B}} \bar{c}=\left\{\mathcal{F}_{\bar{a} \bar{b} \bar{c}}, \mathcal{F}_{\bar{a} \bar{b}} \bar{c}, \mathcal{F}_{\bar{a}}^{\bar{b}} \bar{c}, \mathcal{F}^{\bar{a} \bar{b} \bar{c}}\right\}$ are identified with the fluxes of the T-duality orbit in question. In addition in DFT generalized flux with one index $\mathcal{F}_{A}$ is defined

$$
\begin{aligned}
\mathcal{F}^{\bar{A}}{ }_{\bar{B} \bar{C}} & =2 E_{M}^{\bar{A}} E_{[\bar{B}}^{N} \partial_{N} E_{\bar{C}]}^{M}-E_{M}^{\bar{A}} \eta^{M N} \eta_{K L} \partial_{N} E_{[\bar{B}}^{K} E_{\bar{C}]}^{L}, \\
\mathcal{F}_{\bar{A}} & =\partial_{M} E_{\bar{A}}^{M}+2 E_{\bar{A}}^{M} \partial_{M} d .
\end{aligned}
$$

Using this we have for the backgrounds of $\mathrm{H}$ - and KK-monopoles

H-monopole:

$$
\mathcal{F}_{\bar{z} \bar{a} \bar{b}}=2 e^{z} e^{k} e_{\bar{a}} e_{\bar{b}}^{l} \partial_{[k} A_{l]}, \quad \mathcal{F}^{\bar{a}} \bar{b}_{\bar{c}}=-\delta^{\bar{a}}{ }_{[\bar{b}} \mathfrak{f}_{\bar{c}]}, \quad \mathcal{F}_{\bar{a}}=\frac{3}{2} H^{-1} \partial_{\bar{a}} H,
$$

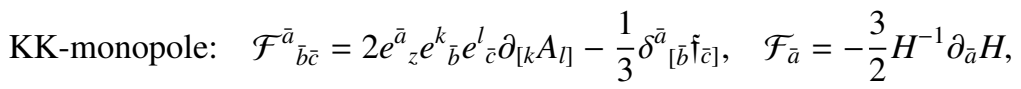

with $(\bar{a}, \bar{b}, \bar{c})$ running from 1 to 3 .

Note that for the above cases the harmonic function $H=H\left(y^{1}, y^{2}, y^{3}\right)$ depends only on physical coordinates and hence the story does not differ much from the conventional supergravity. In contrast, for the non-geometric backgrounds of Q- and R-monopoles $H$ becomes a function of dual coordinates, that is an expected behavior given contributions from worldsheet instantons [24-26]. From the technical side this is crucial for reproducing non-geometric fluxes, whose definition includes derivatives with respect to dual coordinates. 


\subsection{Q- and R-monopoles}

As follows from the Table 2 choosing the physical coordinates as $\left(x^{z}, x^{1}, x^{2}, x^{3}\right)=\left(\tilde{z}, y^{1}, y^{2}, \tilde{y}^{3}\right)$ we obtain the background of Q-monopole

$$
\begin{aligned}
d s^{2} & =\eta_{r s} d x^{r} d x^{s}+H^{-1}\left[\left(d \tilde{z}+A_{\theta} d \theta\right)^{2}+d \tilde{y}_{3}^{2}\right]+H\left(d \rho^{2}+\rho^{2} d \theta^{2}\right), \\
A_{\theta} & =h\left(1-\frac{y^{3}}{\sqrt{\rho^{2}+\left(y^{3}\right)^{2}}}\right), \quad e^{-2\left(\varphi-\varphi_{0}\right)}=H^{-1}, \quad B^{(2)}=0 \\
\rho^{2} & =\left(y^{1}\right)^{2}+\left(y^{2}\right)^{2},
\end{aligned}
$$

where the polar coordinates $(\rho, \theta)$ in the physical plane $\left(x^{1}, x^{2}\right)$ have been adopted. Interestingly, the solution is purely metric, i.e. it does not have non-trivial 2-form or bivector fields similarly to the KKmonopole. However, it still carries non-geometric Q-flux, thas is justified by calculating components of the generalized flux

$$
\begin{aligned}
& Q_{\bar{\theta}}^{\overline{3} \bar{z}}=-H^{-\frac{3}{2}} \partial_{\rho} H, \quad \mathcal{F}_{\bar{\alpha}}^{\bar{\beta} \overline{3}}=-\frac{1}{2} \delta^{\bar{\beta}}{ }_{\bar{\alpha}} H^{-\frac{3}{2}} \tilde{\partial}^{3} H \\
& \text { Q-monopole: } \quad \mathcal{F}^{\bar{z}}{ }_{\bar{\rho} \bar{z}}=\mathcal{F}^{\overline{3}}{ }_{\bar{\rho} \overline{3}}=-\mathcal{F}^{\bar{\theta}}{ }_{\overline{1} \overline{2}}=\frac{1}{3} \mathcal{F}_{\bar{\rho}}=\frac{1}{2} H^{-\frac{3}{2}} \partial_{\rho} H \\
& \mathcal{F}_{\bar{z}}^{\bar{z} \overline{3}}=\frac{1}{3} \mathcal{F}^{\overline{3}}=\frac{1}{2} \mathcal{F}^{\bar{z}}{ }_{\overline{1} \overline{2}}=\frac{1}{2} H^{-\frac{3}{2}} \tilde{\partial}^{3} H,
\end{aligned}
$$

where one notes that $\tilde{\partial}^{3}=\partial / \partial_{\tilde{x}_{3}}$ is the derivative along a winding mode coordinate, the indices $\{\alpha, \beta\}$ run through $\{1,2\}$ and the barred indices are flat indices.

We see, that the background indeed carries a portion of Q-flux and does not carry non-trivial components of other fluxes. Note that the harmonic function has always the same form (17), and for this background it becomes a function of the dual coordinate $\tilde{x}_{3}:=y^{3}$.

For the background of R-monopole the harmonic function becomes a function of two dual coordinates $\tilde{x}_{1}:=y^{1}, \tilde{x}_{2}:=y^{2}$, and the background fields become

$$
\begin{aligned}
d s^{2} & =\eta_{r s} d x^{r} d x^{s}+H^{-1}\left(d \tilde{z}^{2}+d \rho^{2}+\rho^{2} d \theta^{2}\right)+H\left(d y^{1}\right)^{2}, \\
\beta^{\theta z} & =A_{\theta}, \quad e^{-2\left(\varphi-\varphi_{0}\right)}=1 . \\
A_{\theta} & =h\left(1-\frac{y^{1}}{\sqrt{\tilde{\rho}^{2}+\left(y^{1}\right)^{2}}}\right), \quad \rho^{2}=\left(\tilde{y}_{2}\right)^{2}+\left(\tilde{y}_{3}\right)^{2}=\left(x^{2}\right)^{2}+\left(x^{3}\right)^{2} .
\end{aligned}
$$

It is important to note the different meaning of the coordinates $\rho$ and $\tilde{\rho}$, with the latter defined as

$$
\begin{aligned}
& \tilde{x}_{2}=y^{2}=\tilde{\rho} \cos \tilde{\theta}, \\
& \tilde{x}_{3}=y^{3}=\tilde{\rho} \sin \tilde{\theta}, \\
& x^{1}=y^{1} .
\end{aligned}
$$

With that notations in hands components of the generalized flux can be written in the following form

$$
\begin{aligned}
R^{\bar{\alpha} \bar{\alpha}} & =\epsilon^{\bar{\alpha} \bar{\beta}} H^{-\frac{3}{2}} \partial_{1} H, & \mathcal{F}_{\bar{z}} \overline{\bar{\alpha}} \bar{\alpha} & =\mathcal{F}_{\overline{1}}{ }^{\overline{1} \bar{\alpha}}=-\frac{1}{2} H^{-1} \tilde{\partial}^{\bar{\alpha}} H, \\
\text { R-monopole: } \mathcal{F}_{\bar{a}}{ }^{\bar{b} \bar{c}} & =\delta_{1}{ }^{\left[{ }^{[} \bar{b}\right.} \delta_{\bar{a}}{ }^{\bar{c}]} H^{-\frac{3}{2}} \partial_{1} H, & \mathcal{F}_{\bar{\alpha}}{ }^{\bar{\beta} \bar{\gamma}}=\frac{1}{2} \epsilon^{\bar{\beta} \bar{\gamma}} \epsilon_{\bar{\alpha} \bar{\delta}} H^{-1} \tilde{\partial}^{\bar{\delta}} H, & \mathcal{F}_{\overline{1}}{ }^{\bar{z} \bar{\alpha}}=\epsilon^{\bar{\alpha} \bar{\beta}} H^{-1} \tilde{\partial}^{\bar{\beta}} H \\
\mathcal{F}^{\bar{\alpha}} & =\frac{3}{2} H^{-1} \tilde{\partial}^{\bar{\alpha}} H & \mathcal{F}_{\overline{1}} & =\frac{3}{2} H^{-\frac{3}{2}} \partial_{1} H,
\end{aligned}
$$


where $\alpha, \beta$ now take the values 2 and 3. One notices that the $\mathrm{R}$-flux itself is written in terms of derivative of the harmonic function along the physical coordinate, that is the consequence of using the $\beta$-frame of DFT. In addition one finds components of the generalized flux, which as in the previous case, are proportional to derivatives of the harmonic function with respect to dual coordinates.

In principle, one is able to obtain the above results in the B-frame as well, however, we found these calculations too lengthy.

\section{Conclusions}

This letter briefly describes the result of [17] where the solutions of Double Field Theory characterised by non-geometric Q- and R-fluxes have been constructed as particular cases of the DFT-monopole of Berman and Rudolph [27]. By making use of the notion of generalized flux it is explicitly shown, that these solutions indeed carry a portion of Q-flux or R-flux, which satisfy generalized Bianchi identities of [28].

The most interesting feature of these solutions is that the fields depend on dual coordinates. Although, this is an expected behaviour due to worldsheet instanton corrections, one should no forget, that DFT is a low energy field theory and does not have to know about stringy effects. The described effect however gives a hint, that it actually may know about strings more, than conventional supergravity. Performing smearing procedure along these dual coordinates one obtains the known $5_{2}^{2}$-brane solution of [20] from Q-monopole and a co-dimension-1 solution from R-monopole. For both of these the harmonic function becomes not well defined at spatial infinity and hence the solutions are pathological.

In contrast in DFT the solutions behave well at infinity, that allows to define and explicitly calculate magnetic charge, that reads

$$
4 \pi \mu=\int_{\Sigma} \mathcal{F}_{M N K} d \mathbb{X}^{M} \wedge d \mathbb{X}^{N} \wedge d \mathbb{X}^{K}
$$

Here the integration is performed along a special 3-cycle $\Sigma \equiv \mathbb{S}^{2} \times \mathbb{S}^{1}$ which is defined differently for the backgrounds. However, for all the solutions considered in this paper such defined magnetic charge gives the same result $\mu=Q_{N S 5}$, that is just the magnetic charge of the NS5-brane (without smearing). This is expected as the initial definition is $O(d, d)$-invariant.

Table 3. The 3-cycle $\Sigma \equiv \mathbb{S}^{2} \times \mathbb{S}^{1}$ is a product of a 1-circle and a 2-sphere. Here the bullet $\bullet$ denotes the direction of the 1-circle, while the crosses $\times$ denote the directions in which the 2-sphere lives.

\begin{tabular}{|c|cccccccc|}
\hline$\Sigma$ & $x^{1}$ & $x^{2}$ & $x^{3}$ & $x^{z}$ & $\tilde{x}_{1}$ & $\tilde{x}_{2}$ & $\tilde{x}_{3}$ & $\tilde{x}_{z}$ \\
\hline $\mathrm{H}$ & $\times$ & $\times$ & $\times$ & $\bullet$ & & & & \\
$\mathrm{KK}$ & $\times$ & $\times$ & $\times$ & & & & & $\bullet$ \\
$\mathrm{Q}$ & $\times$ & $\times$ & & & & & $\times$ & $\bullet$ \\
$\mathrm{R}$ & $\times$ & & & & & $\times$ & $\times$ & $\bullet$ \\
\hline
\end{tabular}

The main idea behind such defined integration surface is (i) that it cannot vary both in a physical and the corresponding dual directions, that follows from the section condition, (ii) the rest is just chosen by the generalized flux itself and one has nothing else to write.

Note that the coordinates $x^{z}$ or $\tilde{x}_{z}$ are always compact as the fields does not depend on them. In general one may consider the full solution that depends on (one of) these coordinates and to obtain similar results. Such solution of the DFT equations of motion has already been constructed in [27] and called the localized DFT monopole. 


\section{Acknowledgements}

The authors' attendance of the conference QUARKS'16 was sponsored by the Alexander von Humboldt foundation. This work was supported by the Alexander von Humboldt Foundation and in part by the Russian Government programme of competitive growth of Kazan Federal University.

\section{References}

[1] A.A. Tseytlin, Phys.Lett. B242, 163 (1990)

[2] W. Siegel, Phys.Rev. D48, 2826 (1993), hep-th/9305073

[3] N. Hitchin, Lectures on generalized geometry (2010), arXiv: 1008.0973

[4] O. Hohm, C. Hull, B. Zwiebach, JHEP 1007, 016 (2010), 1003.5027

[5] O. Hohm, C. Hull, B. Zwiebach, JHEP 1008, 008 (2010), 1006.4823

[6] D.S. Berman, M.J. Perry (2010), 1008. 1763

[7] D.S. Berman, H. Godazgar, M.J. Perry, Phys.Lett. B700, 65 (2011), 1103.5733

[8] D.S. Berman, H. Godazgar, M. Godazgar, M.J. Perry, JHEP 1201, 012 (2012), 1110.3930

[9] O. Hohm, H. Samtleben, Phys.Rev. D89, 066016 (2014), 1312.0614

[10] O. Hohm, H. Samtleben, Phys.Rev. D89, 066017 (2014), 1312.4542

[11] O. Hohm, H. Samtleben, Phys.Rev. D90, 066002 (2014), 1406. 3348

[12] A. Abzalov, I. Bakhmatov, E.T. Musaev, JHEP 06, 088 (2015), 1504.01523

[13] I. Jeon, K. Lee, J.H. Park, Phys.Rev. D85, 081501 (2012), 1112.0069

[14] M. Cederwall (2016), 1603.04684

[15] H. Godazgar, M. Godazgar, O. Hohm, H. Nicolai, H. Samtleben, JHEP 1409, 044 (2014), 1406.3235

[16] E. Musaev, H. Samtleben, JHEP 1503, 027 (2015), 1412.7286

[17] I. Bakhmatov, A. Kleinschmidt, E.T. Musaev (2016), 1607.05450

[18] J. Shelton, W. Taylor, B. Wecht, JHEP 0510, 085 (2005), hep-th/0508133

[19] D. Andriot, A. Betz, JHEP 07, 059 (2014), 1402 . 5972

[20] J. de Boer, M. Shigemori (2012), 1209.6056

[21] T. Ortin, Gravity and Strings, Cambridge Monographs on Mathematical Physics (Cambridge University Press, 2015), ISBN 9780521768139, 9780521768139

[22] D.S. Berman, F.J. Rudolph, JHEP 05, 015 (2015), 1409.6314

[23] D. Lust, JHEP 12, 084 (2010), 1010. 1361

[24] J.A. Harvey, S. Jensen, JHEP 10, 028 (2005), hep-th/0507204

[25] S. Jensen, JHEP 1107, 088 (2011), 1106. 1174

[26] T. Kimura, S. Sasaki, JHEP 08, 126 (2013), 1305 . 4439

[27] D.S. Berman, D.C. Thompson (2013), 1306. 2643

[28] D. Geissbuhler, D. Marques, C. Nunez, V. Penas (2013), 1304.1472 